\title{
Neurobiologische Grundlagen der Placebo-Analgesie
}

\author{
Falk Eippert
}

In den letzten 40 Jahren wurden die neurobiologischen Mechanismen analgetischer Placeboeffekte eindringlich erforscht. Dank bahnbrechender Studien ist heute bekannt, welche Botenstoffe bei diesem Phänomen wirken und welche Areale des Zentralnervensystems eine tragende Rolle spielen. Trotz dessen sucht die Forschung weiterhin nach Antworten auf etliche offene Fragen - in der Hoffnung, diese Aspekte im klinischen Alltag nachhaltig nutzen zu können.

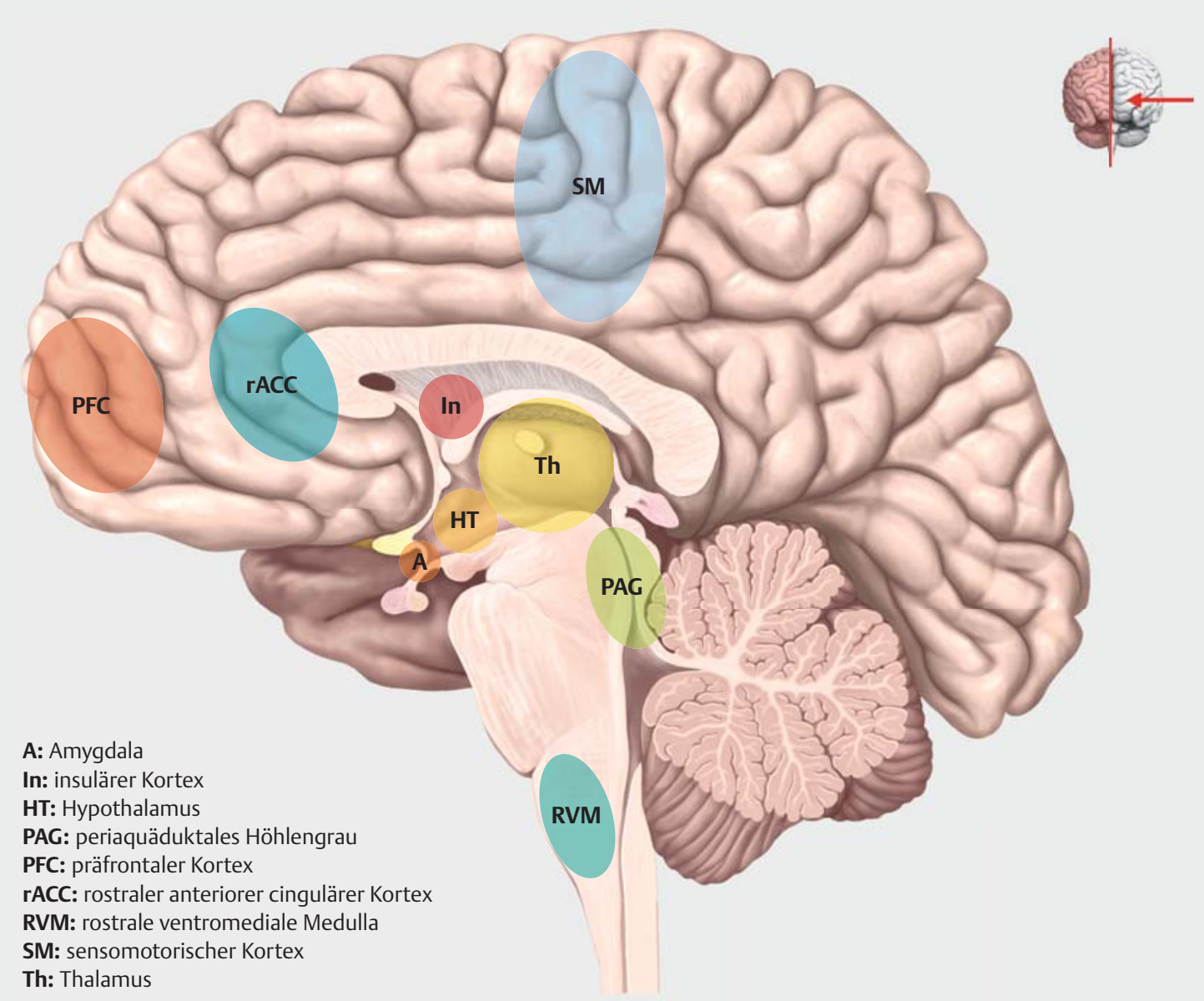

Quelle: Schünke M, Schulte E, Schumacher U. Prometheus. LernAtlas der Anatomie. Kopf, Hals und Neuroanatomie. Illustrationen von K. Wesker. 4. Aufl. Stuttgart: Thieme; 2015. Modifiziert durch Thieme Gruppe.

Schmerz ist eine subjektive Erfahrung - ein Erlebnis, das nicht zwingend in engem Zusammenhang mit der Intensität einer Gewebeverletzung stehen muss. Dieses Phä- nomen ist oftmals bei Sportlern zu beobachten, die während eines Wettkampfes trotz einer starken Verletzung keine Schmerzen verspüren, oder bei chronischen 
Schmerzpatienten, deren Beschwerden ohne eine akute Gewebeverletzung persistieren. Solche grundlagenwissenschaftlich und klinisch interessanten Zustände können auch experimentell erzeugt werden. Die PlaceboAnalgesie, d. h. die durch ein Scheinmedikament verminderte Schmerzempfindung, ist hierfür ein prägnantes Beispiel.

\section{Geschichte der Placebo-Forschung}

Placeboeffekte findet man in unterschiedlichsten Feldern der Medizin - im klinischen Alltag als Reaktion auf die Gabe von Scheinmedikamenten oder in randomisierten klinischen Studien als Kontrollgröße; Placeboeffekte dienen aber auch selbst als Forschungsgegenstand.

"The powerful placebo“ Die wissenschaftliche Beschreibung von Placeboeffekten begann mit dem wegbereitenden Artikel „The powerful placebo“ von Henry K. Beecher im Jahr 1955 [1]. Die neurobiologische PlaceboForschung ist hingegen jüngeren Datums. Erst im Jahr 1978 führten Jon D. Levine et al. erste psychopharmakologische Placebo-Studien in den USA durch [2]. Diese Anstrengungen erfuhren in den 1990er-Jahren durch eine Reihe bedeutender psychopharmakologischer Arbeiten des Italieners Fabrizio Benedetti einen starken Aufschwung [3]. Seit der Jahrtausendwende wird die Forschung v. a. auch durch bildgebende Studien des Gehirns geprägt [4].

Bevor detailliert auf die Ergebnisse dieser und anderer Studien eingegangen wird, sei zunächst auf die weiterführende Literatur verwiesen [5-7]. Des Weiteren werden kurz zwei Themen behandelt, deren Kenntnis für die neurobiologischen Grundlagen der Placebo-Analgesie unabdingbar sind: Methoden der kognitiven Neurowissenschaften sowie die Neuroanatomie der Schmerzverarbeitung.

\section{Methoden der kognitiven Neurowissenschaften}

In diesem Artikel werden Forschungsergebnisse der kognitiven Neurowissenschaften vorgestellt, welche mittels funktioneller Magnetresonanztomographie (fMRT), Positron-Emissions-Tomographie (PET), Elektroenzephalographie (EEG) und Magnetoenzephalographie (MEG) gewonnen wurden.
Messtechniken Während EEG und MEG die elektrische Aktivität der Neuronen direkt an der Kopfoberfläche messen, geschieht dies bei fMRT und PET nur indirekt. Die fMRT registriert Veränderungen des Blutsauerstoffgehalts; die PET misst entweder Blutflussveränderungen oder mittels spezieller Liganden die Bindung von Botenstoffen an bestimmte Reizempfänger wie z. B. Opioid-Rezeptoren. Auch diese Prozesse sind mit einer elektrischen Aktivität von Nervenzellen gekoppelt.

Vor- und Nachteile Die fMRT und die PET sind angesichts einer zeitlichen Auflösung von Sekunden bzw. Minuten der EEG und der MEG mit einer Auflösung im Bereich von Millisekunden zwar deutlich unterlegen, sie bieten aber den unschätzbaren Vorteil, dass ihre räumliche Auflösung im Bereich von Millimetern liegt, wodurch eine relativ präzise Lokalisation von neurobiologischen Prozessen möglich wird.

\section{Neuroanatomie der Schmerzverarbeitung}

Intuitiv glauben die meisten Menschen an das kartesianische Schmerzmodell. Sie denken, dass die neurobiologische Schmerzverarbeitung mit einer Verletzung in der Körperperipherie beginnt und mit der Ankunft von Schmerzsignalen im Großhirn endet. Allerdings ist diese „anatomische Einbahnstraße“ kein exaktes Abbild des neurobiologischen Geschehens. Tatsächlich umfasst die Schmerzverarbeitung neben einem zum Kortex aufsteigenden Ast auch einen vom Gehirn in die Peripherie absteigenden Ast ( $\triangleright$ Abb. 1).

\section{Aufsteigender Ast}

Nach einer Verletzung erreichen nozizeptive Impulse aus dem peripheren Nervensystem das Hinterhorn des Rückenmarks, wo bereits erste komplexe Verarbeitungsschritte stattfinden [8]. Vom Hinterhorn werden die Schmerzsignale dann über verschiedene Pfade zu Strukturen des Hirnstamms, des Mittelhirns wie dem periaquäduktalen Höhlengrau (PAG) sowie Teilen des Zwischenhirns wie der Amygdala und den Thalamus gesendet [9]. Auch hier werden die Schmerzinformationen weiterverarbeitet, bevor sie diverse Areale der Großhirnrinde erreichen - so u.a. die somatosensorischen Kortex-Areale, den insulären Kortex oder den anterioren cingulären Kortex [10]. 


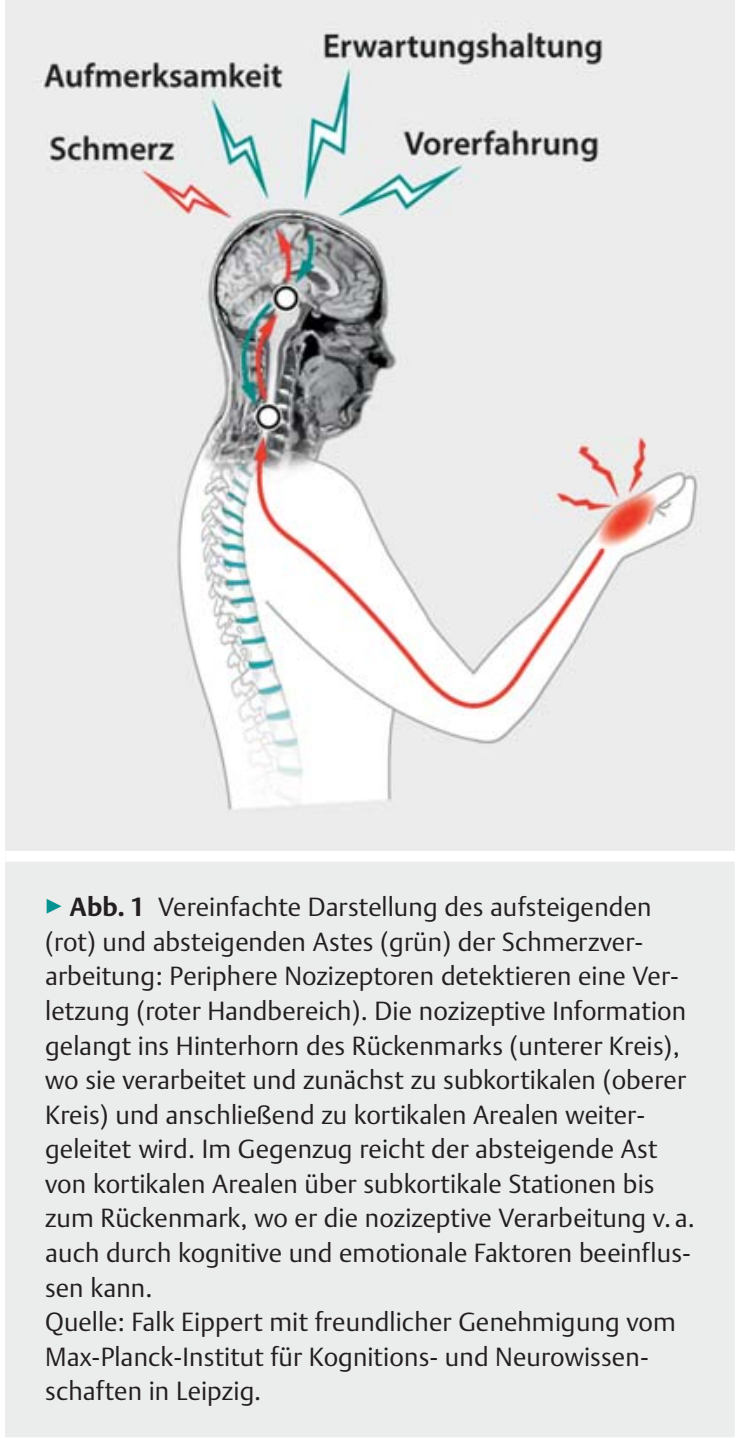

\section{Absteigender Ast}

Neben dem aufsteigenden gibt es auch einen absteigenden Ast der Schmerzverarbeitung. Dieser reicht von der Großhirnrinde bis zum Rückenmark und kann die dortigen spinalen Prozesse und dadurch letztendlich auch die Schmerzwahrnehmung signifikant beeinflussen. Dem absteigenden Ast wurde nicht zuletzt durch die Publikation der ,Gate-Control-Theory، von Ronald Melzack und Patrick David Wall im Jahr 1965 starke Aufmerksamkeit zuteil [11].

\section{PAG-RVM-Schmerzkontrollsystem}

Später kristallisierte sich in Tierversuchen heraus, dass das Herzstück dieses absteigenden Systems aus dem PAG und seinen Verbindungen zur rostralen ventromedialen Medulla (RVM) im Hirnstamm besteht [12]. Im PAG-RVM-System können nozizeptive Verarbeitungsschritte auf Rückenmarksebene sowohl verstärkt wie auch abgeschwächt werden.
Emotionale und kognitive Einflüsse Weitere Untersuchungen machten deutlich, dass dieses Schmerzkontrollsystem durch Nervenzellen des Hypothalamus, der Amygdala und des präfrontalen Kortex kontrolliert werden kann. Es ist damit ideal positioniert, um emotionale und kognitive Einflüsse auf die Schmerzverarbeitung zu realisieren.

\section{Chemische Botenstoffe}

\section{Endogene Opiode}

Parallel zur Erforschung des PAG-RVM-Schmerzkontrollsystems wurden im menschlichen Gehirn Opiat-ähnliche Substanzen entdeckt. Diese endogenen Opioide werden umgangssprachlich auch als „Endorphine“ bezeichnet.

Geburtsstunde Da das PAG-RVM-System endogene Opioide als chemische Botenstoffe bzw. Neurotransmitter zur Schmerzhemmung nutzt, stellten sich US-amerikanische Wissenschaftler die Frage, ob auch die Placebo-Analgesie über Endorphine vermittelt sein könnte [2]. Levine et al. testeten dies an Patienten mit postoperativen Schmerzen. Sie induzierten einen Placeboeffekt, wobei sie einem Teil der Patienten den Opiat-Antagonisten Naloxon verabreichten, also einen Stoff, der die Funktion des endogenen Opioidsystems hemmt: Wäre der Placeboeffekt tatsächlich Opioid-vermittelt, sollten diese Patienten keinen oder einen signifikant schwächeren Placeboeffekt zeigen. Dies war tatsächlich der Fall. Die Entdeckung von Levine et al. wird gemeinhin als die Geburtsstunde der neurobiologischen Erforschung von Placeboeffekten angesehen.

\section{Weitere Neurotransmitter}

Mittlerweile ist die Existenz Opioid-vermittelter Placeboeffekte durch zahlreiche Studien etabliert. Die Wissenschaft fokussiert sich daher auch zunehmend auf die Rolle von anderen Neurotransmittern, die unter bestimmten Rahmenbedingungen ebenfalls Placeboeffekte vermitteln können.

\section{Cholecystokinin}

In zwei Studien konnten Benedetti et al. zeigen, dass der Botenstoff Cholecystokinin (CCK) gewissermaßen als Anti-Opioid funktioniert. CCK vermittelt nicht nur Noceboeffekte bzw. eine Verstärkung der Schmerzen, sondern CCK-Hemmer können auch Placeboeffekte verstärken $[3,13]$.

\section{Endocannabinoide}

Auch körpereigene Cannabinoide bzw. Endocannabinoide sind offensichtlich bei der Vermittlung von Opioidunabhängigen Placeboeffekten beteiligt [14]. Die diesbezügliche Forschung steht allerdings erst am Anfang. 

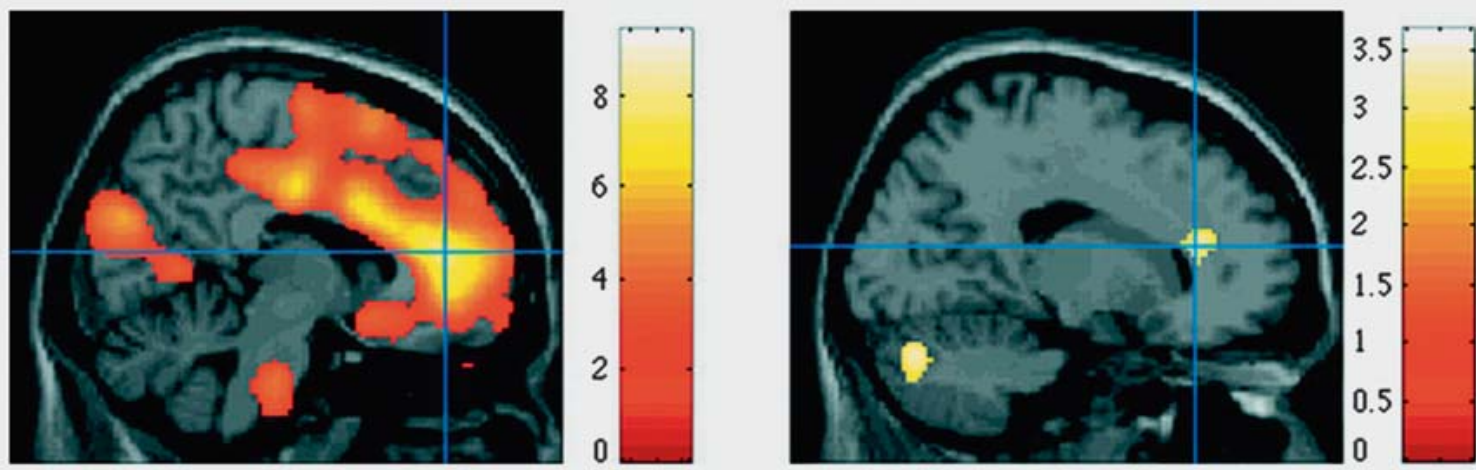

- Abb. 2 Kortikale Schmerzmodulation: Nach Gabe von Remifentanil wird im Gehirn der rostrale anteriore cinguläre Kortex (rACC) aktiviert (linkes Fadenkreuz). Wird anstatt des hochpotenten Opiats ein Placebo verabreicht, zeigt die PET ebenfalls eine signifikante Aktivierung des rACC (rechtes Fadenkreuz). Die jeweilige Stärke der kortikalen Aktivierung ist über die Farbskala kodiert. Quelle: Falk Eippert mit freundlicher Genehmigung von Petrovic et al. (2002) [4]. Reprinted with permission from AAAS.

\section{Dopamin und Oxytocin}

Noch unklar ist die Bedeutung des aus dem Kontext von Morbus Parkinson bekannten Neurotransmitters Dopamin sowie des sog. „Bindungshormons“ Oxytocin. Einerseits gibt es klare Hinweise, dass beide Botenstoffe bei Placeboeffekten involviert sind $[15,16]$. Andererseits liegen Studien vor, welche diese Hypothese nicht stützen $[17,18]$. Infolgedessen ist eine abschließende Bewertung gegenwärtig nicht möglich.

\section{Kortikale Schmerzmodulation}

Wenngleich die Identifikation der bei einem Placeboeffekt beteiligten Neurotransmitter von enormer Bedeutung ist, sagt dies - aufgrund der weiträumigen Verteilung dieser Botenstoffe im Nervensystem - jedoch noch nichts darüber aus, welche Hirnareale einen Beitrag zur Placebo-Analgesie leisten.

\section{Rostraler anteriorer cingulärer Kortex}

Im Jahr 2002 zeigte eine PET-Studie der schwedischen Forschergruppe um Predrag Petrovic, dass sowohl die Gabe des stark wirksamen Opiats Remifentanil als auch eines Placebo-Präparats zur Schmerzreduktion führte und in beiden Fällen der rostrale anteriore cinguläre Kortex (rACC) aktiviert wurde - eine Hirnregion, die reich an Opiat-Rezeptoren ist [4] (ฉ Abb. 2).

Drei Jahre später bestätigten Jon-Kar Zubieta und sein Team von der Universität Michigan diesen ersten indirekten Hinweis auf einen lokalen Effekt endogener Opioide unter Placebo. Mittels Opioid-sensitiver PET wiesen sie nach, dass unter Placebo eine erhöhte Ausschüttung von Endorphinen im rACC stattfindet [19]. Inzwischen wurde die zentrale Rolle des rACC bei Placeboeffekten durch Metaanalysen bestätigt [20].

\section{Präfrontaler Kortex}

Der rACC ist nicht die einzige kortikale Region, die bei Placeboeffekten von Bedeutung ist. Bis dato konnten mehrere Studien belegen, dass auch präfrontale KortexRegionen eine verstärkte Aktivierung unter Placebo zeigen [21]. Insbesondere handelt es sich um den dorsolateralen präfrontalen Kortex (dIPFC) - ein Hirnareal, das oftmals mit Entscheidungsprozessen sowie der Top-DownRegulation von Wahrnehmung in Verbindung gebracht wird.

Zusätzlich zu diesen korrelativen Ergebnissen konnte die Wichtigkeit des dIPFC auch kausal demonstriert werden. Peter Krummenacher et al. wiesen im Jahr 2009 nach, dass eine Hemmung der Nervenzellaktivität im dIPFC mittels transkranieller Magnetstimulation zur kompletten Blockade der Placebo-Analgesie führt [22].

\section{Verbindungen zum PAG-RVM-System}

Der rACC sowie der dIPFC stehen relativ hoch in der kortikalen Hierarchie und sind v. a. mit affektiven und kognitiven Prozessen assoziiert. Interessanterweise stehen beide Hirnregionen mit dem absteigenden PAG-RVM-System in Verbindung. Demzufolge könnten sie über dieses Schmerzkontrollsystem eine tiefgreifende Modulation der Schmerzwahrnehmung vornehmen. Drei Studien am Uniklinikum in Hamburg Eppendorf (UKE) unterstützen diese Vermutung. 
rACC-PAG-Kopplung Zunächst wiesen Ulrike Bingel et al. im Jahr 2006 nach, dass die funktionelle Kopplung von rACC und PAG unter Placebo signifikant erhöht ist [23]. Drei Jahre später demonstrierten Falk Eippert et al., dass die rACC-PAG-Kopplung die individuelle Ausprägung von Placeboeffekten sowie die RVM-Aktivierung vorhersagen kann [24]. Niklas Stein et al. demonstrierten schließlich im Jahr 2012, dass die Stärke der strukturellen Verbindungen zwischen rACC und PAG als Prädiktor für die individuelle Ausprägung von Placeboeffekten geeignet ist [25].

\section{Interpretation}

Die drei UKE-Studienergebnisse könnten dahingehend interpretiert werden, dass Placebo-Analgesie mittels der Erwartung von Schmerzreduktion zur Aktivierung des PAG-RVM-Systems führt. Dieses wiederum hemmt bereits auf Rückenmarksebene die nozizeptive Verarbeitung und führt somit zur verminderten Weiterleitung der Schmerzsignale zu den subkortikalen und kortikalen Regionen. Für diese Annahme spricht u. a. auch ein Befund, der über verschiedenste fMRT-Studien hinweg berichtet wurde $[21,23,24,26]$. Und auch die Metaanalysen von Karin Meissner et al. sowie die eindrucksvollen MEG-Ergebnisse von Jürgen Lorenz et al. bestätigen, dass unter Placebo-Analgesie eine verminderte Aktivierung von Hirnregionen zu beobachten ist, die traditionell mit der Schmerzverarbeitung in Verbindung gebracht werden $[20,27]$. Diese sind u. a. der Thalamus, die somatosensorischen Kortex-Areale, der anteriore cinguläre Kortex sowie der insuläre Kortex. Die dort nachgewiesene reduzierte Aktivität könnte ein Grund für die verminderte Schmerzempfindung unter Placebo sein.

\section{Spinale Schmerzmodulation}

Um die Hypothese zu verifizieren, dass es unter PlaceboAnalgesie zur spinalen Hemmung der nozizeptiven Verarbeitung kommt, ist die alleinige Interpretation kortikaler fMRT-Befunde nicht ausreichend. Idealerweise müssen direkt die Prozesse im menschlichen Rückenmark gemessen werden, um zu prüfen, ob es hier unter PlaceboAnalgesie zu Veränderungen kommt.

\section{Indirekte Messung}

Dies wurde erstmalig im Jahr 2006 erfolgreich von Dagfinn Matre und Kollegen realisiert [28]. Mittels eines eleganten Paradigmas induzierten sie bei ihren Probanden eine starke Placebo-Analgesie auf eine Hitzeschmerzstimulation. Als Maß der spinalen Verarbeitung nutzten sie die Ausbreitung der sekundären Hyperalgesie und der Allodynie - beides bekanntermaßen spinal vermittelte Prozesse. Im Fokus der Wissenschaftler aus Norwegen stand also die Ausdehnung des Hautareals um den applizierten Hitzestimulus, in dem die Verabreichung nicht-schmerzhafter Reize dennoch Schmerzen bei den Probanden auslöste. Matre et al. konnten unter der Place-
bo-Bedingung eine signifikant kleinere räumliche Ausbreitung der Hyperalgesie sowie der Allodynie beobachten und erbrachten damit den ersten Nachweis für spinale Veränderungen unter Placebo-Analgesie.

Die Placebo-Analgesie gehört folglich zu einer Reihe von Phänomenen der Schmerzmodulation, bei denen eine spinale Komponente existiert wie z.B. bei Manipulationen der Aufmerksamkeit, des affektiven Kontextes sowie der Erwartungshaltung.

\section{Direkte Messung}

Während Matre et al. nur ein indirektes Maß für die spinale Verarbeitung nutzen konnten, ist es seit einigen Jahren möglich, mittels der fMRT direkt die Aktivität des Rückenmarks zu messen und zu kontrollieren, ob die spinalen Antworten auf Schmerzreize unter Placebo-Analgesie verringert sind. Dies ist allerdings u. a. aufgrund der anatomischen Lage und der Struktur des Rückenmarks sowie dem störenden Einfluss von Herzschlag und Atmung methodisch schwierig.

Im Jahr 2009 beobachteten Eippert et al., dass neben einer reduzierten Schmerzempfindung in der PlaceboBedingung auch verringerte Antworten im ipsilateralen Hinterhorn des Rückenmarks zu finden sind - d. h. genau an jener Stelle, die im Normalzustand die stärksten Schmerzantworten zeigt [29] ( $\bullet$ Abb. 3).

Dieser Befund einer direkten Messung der Veränderung spinaler Antworten konnte mittlerweile in verschiedenen Formen der Schmerzmodulation gezeigt werden wie bspw. einer Hemmung unter Ablenkung oder einer Verstärkung im Kontext einer Nocebo-Hyperalgesie [30,31].

Zusammengefasst sprechen diese Ergebnisse dafür, dass die Hemmung nozizeptiver Impulse auf Rückenmarksebene ein Mechanismus ist, welcher die verringerte Schmerzempfindung unter Placebo-Analgesie möglicherweise erklären kann. Allerdings existieren diesbezüglich auch gegenteilige Interpretationen, die rein kortikalen und affektiven Prozessen mehr Gewicht geben [3234].

\section{Fazit und Ausblick}

In der Wissenschaft wurden seit den ersten pharmakologischen Placebo-Studien Ende der 1970er-Jahre erstaunliche Fortschritte gemacht, um das Faszinosum der Placebo-Analgesie durch neurobiologische Prozesse zu erklären. So sind heute nicht nur bestimmte Transmittersysteme als chemische Vermittler der Placebo-Analgesie, sondern auch die Regionen des zentralen Nervensystems vom Großhirn bis hinab zum Rückenmark bekannt, die bei diesem subjektiven Phänomen eine tragende Rolle spielen. Trotz dessen geht die Forschung weiter, und es finden sich in der aktuellen Literatur nach wie vor sowohl 

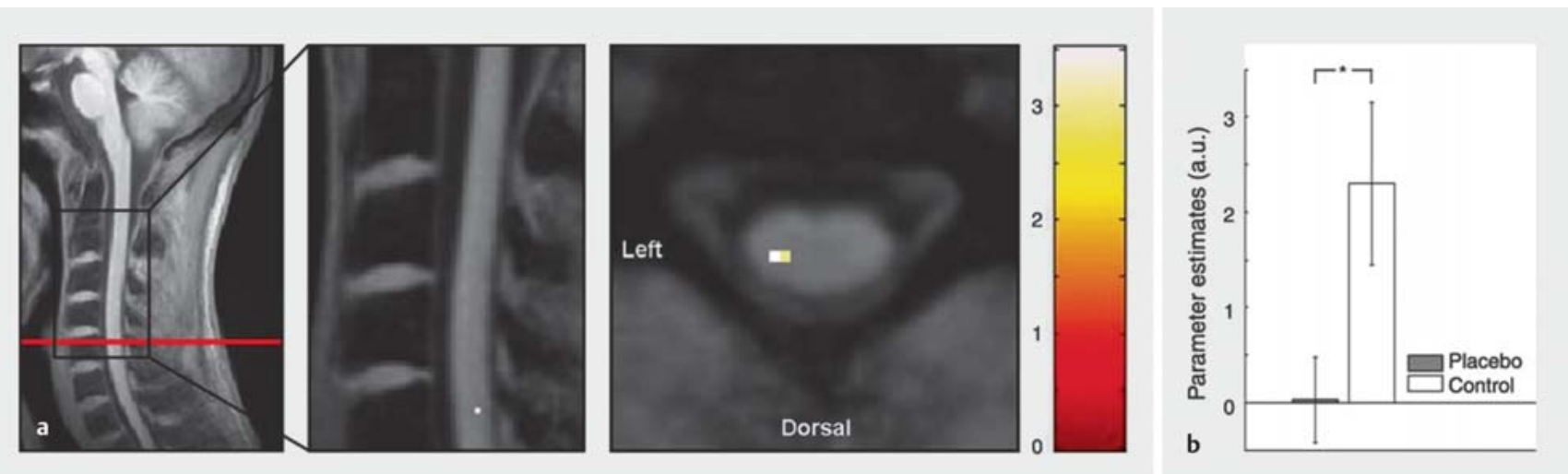

- Abb. 3 Spinale Schmerzmodulation: Sagittalschnitt durch die HWS (links) mit Vergrößerung (Mitte) und Transversalschnitt in Höhe der roten Linie (rechts). Die spinale Aktivierung auf eine Hitzeschmerzstimulation des linken Unterarms imponiert ipsilateral im dorsalen Teil des Rückenmarks (gelb-weiß). Beim Vergleich der Stärke der Rückenmarksantworten unter einer Kontrollbedingung mit einer Placebo-Bedingung (ganz rechts) zeigt sich, dass die spinale Antwort unter Placebo signifikant reduziert ist.

Quelle: Falk Eippert mit freundlicher Genehmigung aus Eippert et al. (2009) [29]. Reprinted with permission from AAAS.

etliche offene Fragen als auch sehr spannende und innovative Ansätze, die weitere Einsichten in Placeboeffekte versprechen - mit der Hoffnung, diese Aspekte auch im klinischen Alltag nachhaltig nutzen zu können.

\section{Offene Fragen}

Generalisierbarkeit Offen bleibt zunächst die Frage, ob sich die dargestellten Ergebnisse auf alle Menschen übertragen lassen. Tatsächlich beruhen die bisher erhobenen Daten hauptsächlich auf gesunden jungen Erwachsenen. Zwar gibt es Hinweise darauf, dass Placeboeffekte sowohl bei Kindern als auch im fortgeschrittenen Alter in ähnlicher Stärke vorhanden sind, es ist jedoch größtenteils ungeklärt, ob hier die gleichen neurobiologischen Mechanismen zugrunde liegen [35, 36].

Krankheitsbilder Weiterhin ist ungewiss, inwieweit die dargestellten Mechanismen auch in verschiedenen Krankheitsbildern zum Tragen kommen. Nach aktuellen Ergebnissen von Andrea Power et al. legen Verhaltensstudien nahe, dass es bei gesunden Probanden und chronischen Schmerzpatienten zu ähnlich ausgeprägten Placeboeffekten kommen kann [37]. Dessen ungeachtet ist eine systematische Untersuchung der neurobiologischen Prozesse über verschiedene Krankheitsbilder hinweg bis dato kaum erfolgt. Dies wäre hinsichtlich von Schmerzkontrolldefiziten wie z. B. bei der Fibromyalgie höchst interessant.
Methodik Drittens lässt sich diskutieren, ob die aktuelle Forschung mit ihren hauptsächlich korrelativen und nicht-invasiven Methoden der kognitiven Neurowissenschaften nah genug an die interessierenden neurobiologischen Prozesse heranreicht. In diesem Kontext erwähnenswert sind einerseits Studien im Rahmen von Hirnoperationen, die z.B. Einzelnervenzellableitungen mit Placebo-induzierten Verhaltensänderungen in Verbindung bringen, sowie andererseits Placebo-ähnliche Prozeduren in Tiermodellen, die einen direkteren Zugang zu neuronalen Prozessen erlauben würden [38, 39].

\section{Innovative Ansätze}

Abschließend seien noch einige innovative und erst vor kurzem mehr in den wissenschaftlichen Fokus geratene Ansätze vorgestellt, mittels derer zurzeit verschiedene Aspekte der Placebo-Analgesie untersucht werden.

So können Konzepte aus der Lerntheorie sowie der Bayesschen Statistik erklären, wie die bei Placebo-Analgesie existierende Diskrepanz zwischen wahrgenommener Schmerzintensität und tatsächlichem nozizeptivem Input entstehen und aufrechterhalten werden kann [40,41]. Weiterhin erlauben Fortschritte in der Datenverfügbarkeit sowie die Methoden der multivariaten Mustererkennung neue Einsichten in die zugrundliegenden Mechanismen der Placebo-Analgesie [32, 34]. Und schließlich wird auch die Entstehung und Notwendigkeit von Erwartungshaltungen für die Entstehung der Placebo-Analgesie untersucht. Auf der einen Seite beinhaltet dies die Gabe von sog. ,Open-Label-Placebos‘, bei denen keine Täuschung vorliegt und der Patient wissentlich ein Scheinmedikament erhält, auf der anderen Seite Techniken, mittels derer Placeboeffekte ,unbewusst' ausgelöst werden können [42,43]. 


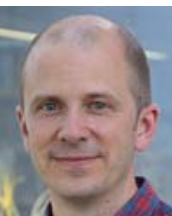

Dr. Falk Eippert leitet die Forschungsgruppe „Schmerzwahrnehmung“ am Max-PlanckInstitut für Kognitions- und Neurowissenschaften in Leipzig. Die Gruppe benutzt bildgebende Verfahren, um neuronale Mechanismen aufzudecken, welche der menschlichen Schmerzwahrnehmung und ihrer kognitiven Modulation zugrunde liegen.

\section{Korrespondenzadresse}

\section{Dr. Falk Eippert}

Max-Planck-Institut für Kognitions- und Neurowissenschaften Stephanstraße 1a

04103 Leipzig

Deutschland

eippert@cbs.mpg.de

\section{Literatur}

[1] Beecher HK. The powerful placebo. JAMA 1955; 159: 1602 1606. doi:10.1001/jama.1955.02960340022006

[2] Levine JD, Gordon NC, Fields HL. The mechanism of placebo analgesia. Lancet 1978; 2: 654-657. doi:10.1016/s0140-6736 (78)92762-9

[3] Benedetti F, Amanzio M, Maggi G. Potentiation of placebo analgesia by proglumide. Lancet 1995; 346: 1231. doi:10.1016/s0140-6736(95)92938-x

[4] Petrovic P, Kalso E, Petersson KM et al. Placebo and opioid analgesia - imaging a shared neuronal network. Science 2002; 295: 1737-1740. doi:10.1126/science. 1067176

[5] Bundesärztekammer. Placebo in der Medizin. Herausgegeben von der Bundesärztekammer auf Empfehlung ihres Wissenschaftlichen Beirats. Köln: Deutscher Ärzte Verlag; 2011. Im Internet unter: https://www.bundesaerztekammer.de/ fileadmin/user_upload/downloads/Placebo_LF_1_17012011. pdf; Stand: 05.08.2020

[6] Brown WA. The placebo effect in clinical practice. London: Oxford University Press; 2013

[7] Benedetti F. Placebo effects: understanding the mechanisms in health and disease. 2. Aufl. London: Oxford University Press; 2014

[8] Todd AJ. Neuronal circuitry for pain processing in the dorsal horn. Nat Rev Neurosci 2010; 11: 823-836. doi:10.1038/ nrn2947

[9] Lima D. Ascending pathways: anatomy and physiology. In: Basbaum A, Bushnell M, Hrsg. Science of Pain. Oxford: Academic Press; 2009

[10] Treede RD, Kenshalo DR, Gracely RH et al. The cortical representation of pain. Pain 1999; 79: 105-111. doi:10.1016/ S0304-3959(98)00184-5

[11] Melzack R, Wall PD. Pain mechanisms: a new theory. Science 1965; 150: 971-979. doi:10.1126/science.150.3699.971

[12] Heinricher MM, Fields HL. Central Nervous System Mechanisms of Pain Modulation. In: McMahon SB, Koltzenburg M, Tracey I et al, Hrsg. Wall and Melzack's textbook of pain. 6. Aufl. Philadelphia, USA: Elsevier Saunders; 2013

[13] Benedetti F, Amanzio M, Vighetti S et al. The biochemical and neuroendocrine bases of the hyperalgesic nocebo effect.
J Neurosci 2006; 26: 12014-12022. doi:10.1523/JNEUROSCI. 2947-06.2006

[14] Benedetti F, Amanzio M, Rosato R et al. Nonopioid placebo analgesia is mediated by CB1 cannabinoid receptors. Nat Med 2011; 17: 1228-1230. doi:10.1038/nm.2435

[15] Scott D], Stohler CS, Egnatuk CM et al. Individual differences in reward responding explain placebo-induced expectations and effects. Neuron 2007; 55: 325-336. doi:10.1016/j.neuron. 2007.06.028

[16] Kessner S, Sprenger C, Wrobel $\mathrm{N}$ et al. Effect of oxytocin on placebo analgesia: a randomized study. JAMA 2013; 310: 1733-1735. doi:10.1001/jama.2013.277446

[17] Wrobel N, Wiech K, Forkmann K et al. Haloperidol blocks dorsal striatum activity but not analgesia in a placebo paradigm. Cortex 2014; 57: 60-73. doi:10.1016/j.cortex.2014.02.023

[18] Skvortsova A, Veldhuijzen DS, van Middendorp $\mathrm{H}$ et al. Effects of Oxytocin on Placebo and Nocebo Effects in a Pain Conditioning Paradigm: A Randomized Controlled Trial. J Pain 2019; 19: 30792-30298. doi:10.1016/j.jpain.2019.08.010

[19] Zubieta J-K, Bueller JA, Jackson LR et al. Placebo effects mediated by endogenous opioid activity on mu-opioid receptors. J Neurosci 2005; 25: 7754-7762. doi:10.1523/JNEUROSCI. 0439-05.2005

[20] Meissner K, Bingel U, Colloca L et al. The placebo effect: advances from different methodological approaches. J Neurosci 2011; 31: 16117-16124. doi:10.1523/JNEUROSCI. 4099-11.2011

[21] Wager TD, Rilling JK, Smith EE et al. Placebo-induced changes in FMRI in the anticipation and experience of pain. Science 2004; 303: 1162-1167. doi:10.1126/science. 1093065

[22] Krummenacher P, Candia V, Folkers $G$ et al. Prefrontal cortex modulates placebo analgesia. Pain 2009; 148: 368-374. doi:10.1016/j.pain.2009.09.033

[23] Bingel U, Lorenz ], Schoell E et al. Mechanisms of placebo analgesia: rACC recruitment of a subcortical antinociceptive network. Pain 2006; 120: 8-15. doi:10.1016/j.pain.2005. 08.027

[24] Eippert F, Bingel U, Schoell ED et al. Activation of the opioidergic descending pain control system underlies placebo analgesia. Neuron 2009; 63: 533-543. doi:10.1016/j.neuron. 2009.07.014

[25] Stein N, Sprenger C, Scholz J et al. White matter integrity of the descending pain modulatory system is associated with interindividual differences in placebo analgesia. Pain 2012; 153: 2210-2217. doi:10.1016/j.pain.2012.07.010

[26] Price DD, Craggs J, Verne GN et al. Placebo analgesia is accompanied by large reductions in pain-related brain activity in irritable bowel syndrome patients. Pain 2007; 127: 63-72. doi:10.1016/j.pain.2006.08.001

[27] Lorenz J, Hauck M, Paur RC et al. Cortical correlates of false expectations during pain intensity judgments-a possible manifestation of placebo/nocebo cognitions. Brain Behav Immun 2005; 19: 283-295. doi:10.1016/j.bbi.2005.03.010

[28] Matre D, Casey KL, Knardahl S. Placebo-induced changes in spinal cord pain processing. J Neurosci 2006; 26: 559-563. doi:10.1523/JNEUROSCI.4218-05.2006

[29] Eippert F, Finsterbusch J, Bingel U et al. Direct evidence for spinal cord involvement in placebo analgesia. Science 2009; 326: 404. doi:10.1126/science.1180142

[30] Sprenger C, Eippert F, Finsterbusch J et al. Attention modulates spinal cord responses to pain. Curr Biol 2012; 22: 10191022. doi:10.1016/j.cub.2012.04.006 
[31] Geuter S, Büchel C. Facilitation of pain in the human spinal cord by nocebo treatment. J Neurosci 2013; 33: 1378413790. doi:10.1523/JNEUROSCI.2191-13.2013

[32] Wager TD, Atlas LY, Leotti LA et al. Predicting individual differences in placebo analgesia: contributions of brain activity during anticipation and pain experience. J Neurosci 2011; 31: 439-452. doi:10.1523/JNEUROSCI.3420-10.2011

[33] Martini M, Lee MCH, Valentini E et al. Intracortical modulation, and not spinal inhibition, mediates placebo analgesia. Eur J Neurosci 2015; 41: 498-504. doi:10.1111/ejn.12807

[34] Zunhammer M, Bingel U, Wager TD et al. Placebo Effects on the Neurologic Pain Signature: A Meta-analysis of Individual Participant Functional Magnetic Resonance Imaging Data. JAMA Neurol 2018; 75: 1321-1330. doi:10.1001/jamaneurol. 2018.2017

[35] Wrobel N, Fadai T, Sprenger $C$ et al. Are Children the Better Placebo Analgesia Responders? An Experimental Approach. J Pain 2015; 16: 1005-1011. doi:10.1016/j.jpain.2015.06.013

[36] Wrobel N, Fadai T, Brassen S et al. Preserved Capacity for Placebo Analgesia in the Elderly. J Pain 2016; 17: 1318-1324. doi:10.1016/j.jpain.2016.08.012

[37] Power A, Brown C, Sivan M et al. Individuals with chronic pain have the same response to placebo analgesia as healthy controls in terms of magnitude and reproducibility. Pain 2020; Online ahead of print. doi:10.1097/j.pain.000000000000 1966

[38] Benedetti F, Colloca L, Torre E et al. Placebo-responsive Parkinson patients show decreased activity in single neurons of subthalamic nucleus. Nat Neurosci 2004; 7: 587-588. doi:10.1038/nn1250

[39] Nolan TA, Price DD, Caudle RM et al. Placebo-induced analgesia in an operant pain model in rats. Pain 2012; 153: 20092016. doi:10.1016/j.pain.2012.04.026

[40] Schenk LA, Sprenger C, Onat S et al. Suppression of Striatal Prediction Errors by the Prefrontal Cortex in Placebo Hypoalgesia. J Neurosci 2017; 37: 9715-9723. doi:10.1523/ JNEUROSCI.1101-17.2017

[41] Grahl A, Onat S, Büchel C. The periaqueductal gray and Bayesian integration in placebo analgesia. Elife 2018; 7: e32930. doi:10.7554/eLife.32930

[42] Kaptchuk T], Friedlander E, Kelley JM et al. Placebos without deception: a randomized controlled trial in irritable bowel syndrome. PLoS ONE 2010; 5: e15591. doi:10.1371/journal. pone. 0015591

[43] Jensen KB, Kaptchuk TJ, Kirsch I et al. Nonconscious activation of placebo and nocebo pain responses. Proc Natl Acad Sci USA 2012; 109: 15959-15964. doi:10.1073/pnas. 1202056109

\section{Bibliografie}

Der Schmerzpatient 2020; 3: 158-165

DOI $10.1055 / \mathrm{a}-1205-5280$

ISSN 2512-6210

(C) 2020. Thieme. All rights reserved.

Georg Thieme Verlag KG, Rüdigerstraße 14, 70469 Stuttgart, Germany 